\title{
Optical coherence tomography in anterior segment imaging
}

\author{
Maya Kalev-Landoy ${ }^{1}$, Alexander C. Day ${ }^{1}$, M. Francesca Cordeiro ${ }^{1,2}$, and Clive Migdal $^{1}$ \\ ${ }^{1}$ Glaucoma Service, The Western Eye Hospital, London, UK \\ ${ }^{2}$ Institute of Ophthalmology, University College London, London, UK
}

\begin{abstract}
Purpose-To evaluate the ability of optical coherence tomography (OCT), designed primarily to image the posterior segment, to visualize the anterior chamber angle (ACA) in patients with different angle configurations.

Methods-In a prospective observational study, the anterior segments of 26 eyes of 26 patients were imaged using the Zeiss Stratus OCT, model 3000. Imaging of the anterior segment was achieved by adjusting the focusing control on the Stratus OCT. A total of 16 patients had abnormal angle configurations including narrow or closed angles and plateau irides, and $10 \mathrm{had}$ normal angle configurations as determined by prior full ophthalmic examination, including slitlamp biomicroscopy and gonioscopy.
\end{abstract}

Results-In all cases, OCT provided high-resolution information regarding iris configuration. The ACA itself was clearly visualized in patients with narrow or closed angles, but not in patients with open angles.

Conclusions-Stratus OCT offers a non-contact, convenient and rapid method of assessing the configuration of the anterior chamber. Despite its limitations, it may be of help during the routine clinical assessment and treatment of patients with glaucoma, particularly when gonioscopy is not possible or difficult to interpret.

\section{Keywords}

glaucoma; imaging; optical coherence tomography

\section{Introduction}

Understanding the anterior chamber angle (ACA) configuration is an essential part of the diagnosis and management of glaucoma patients. Gonioscopy is the gold standard method for clinically assessing the anterior chamber angle (Shaffer \& Tour 1955; Bruno \& Alward 2002). However, historically, gonioscopy has proved a difficult skill to master; it requires clinical experience and expertise for accurate, albeit subjective, interpretation, involves direct contact with the subject's eye, and is dependent on corneal clarity (Bruno \& Alward 2002). Imaging techniques such as ultrasound biomicroscopy (UBM) have been developed to aid the clinical evaluation of the ACA.

Ultrasound biomicroscopy produces high-resolution, quantitative cross-sectional images of the ACA (Riley et al. 1994; Spaeth et al. 1995), but is not readily available, is relatively

\footnotetext{
Copyright $\odot$ Acta Ophthalmol Scand 2007.

Correspondence: Alexander C. Day, Western Eye Hospital, 153-173 Marylebone Road, London NW1 5QH, UK, Tel: + 44207886 3258, Fax: + 44207886 3259, Email: alex@acday.co.uk.
} 
invasive, is time-consuming and requires a highly skilled operator. Although optical coherence tomography (OCT) designed specifically for imaging the ACA is now commercially available, Zeiss Stratus OCT (Carl Zeiss Meditec, Dublin, CA, USA) units used to image the posterior segment are already available in many eye departments. Their ability to visualize the anterior chamber configuration was recently demonstrated in three subjects with impending angle closure, plateau iris configuration and phacomorphic glaucoma, respectively (Leung et al. 2005). Compared with UBM, OCT is a non-invasive, non-contact technique that is quick and easy to use (Puech \& El Maftouhi 2004; Leung et al. 2005; Radhakrishnan et al. 2005a, 2005b; Wirbelauer et al. 2005).

In this study, we investigated the ability of the Zeiss Stratus OCT, model 3000, to image the ACA in patients with different angle configurations.

\section{Materials and Methods}

In a prospective observational study, the ACA was scanned using OCT in 26 eyes of 26 patients. Informed consent was obtained after explanation of the procedure. Sixteen eyes with narrow or closed angles or plateau iris configuration as determined by gonioscopy were compared with 10 eyes with normal open angles (the latter were used as controls). The angle was defined as normal when graded on gonioscopy as 3 or 4 over $\geq 270$ degrees according to the Shaffer grading system (Shaffer \& Tour 1955; Chan et al. 1981). The angle was defined as narrow if it was graded as 1 or 2 over $\geq 270$ degrees. Plateau iris configuration was defined as a near normal anterior chamber depth with a flat iris profile and crowding of the ACA at the iris insertion.

All subjects underwent a full ophthalmic examination including visual acuity, slit-lamp biomicroscopy, applanation tonometry, indentation gonioscopy (using the Posner fourmirror indentation gonioscopy lens) and fundoscopy prior to imaging of the ACA using the Stratus OCT 3000 instrument. In patients who required laser treatment (peripheral iridotomy or iridoplasty), an OCT scan was performed both before and immediately after the laser treatment.

For OCT imaging of the ACA, the focus control on the OCT unit head was adjusted to enable focusing on the limbal area of each patient. A 'line' protocol (usually 3-5 mm) was then selected from the scan menu, using the real-time camera image on the scanning panel. The patient's eye was maintained in the primary position by appropriate positioning of the external fixation target. Once a signal had been detected, an optimal image was obtained using a technique similar to that employed when scanning the retina. Images of the ACA could be recorded at any position by means of adjusting the angle setting such that the scan beam was directed perpendicularly to the limbus. We found visualization of the ACA was easiest for the nasal and temporal regions; for other areas, manipulation of the patient's eyelid was required. Both nasal and temporal images were compared with gonioscopic findings. In all patients, imaging of the ACA using OCT was carried out under standardized lighting conditions. In patients where plateau iris configuration was diagnosed, UBM (P40; Paradigm Medical Industries, Salt Lake City, UT, USA) was performed for comparison.

As current Stratus OCT software does not allow quantitative analysis of the ACA, we were unable to directly compare the findings on gonioscopy with those seen on OCT. Consequently, all patients were classified as having either open angles, narrow angles, closed angles or plateau iris configuration according to the definitions mentioned above.

To quantify the ability of the Stratus OCT to image the anterior chamber, images were assessed using the following criteria: 
- $\quad$ image quality (clarity of images of ocular structure): poor, good, excellent;

- anterior chamber angle:

- demonstration: not demonstrated, partly demonstrated, clearly demonstrated;

- appearance: closed angle, narrow angle, open angle;

- iris configuration: not clearly demonstrated, clearly demonstrated.

\section{Results}

In all 26 cases, OCT provided high-resolution information regarding iris configuration. However there was variable demonstration of the ACA (Table 1) and limited penetration to the ciliary body and scleral spur (Fig. 1A, B). This was not related to the patient's refractive status (Table 1).

In cases where the cornea was too cloudy to enable a clear gonioscopic view, as in acute angle-closure glaucoma, imaging of the angle was still possible with minimal image degradation (Fig. 1C). It was also possible to easily confirm the five cases of plateau iris configuration with the OCT.

Acquiring the images was straight-forward and similarly easy to imaging the posterior segment. All were of satisfactory quality, with minor issues identified, including movement artefacts, that required the scan to be retaken, and limbal calcium deposits (in one patient in the control group) that caused a shadow across part of the scan. Although some patients in the control group were wearing soft contact lenses when the OCT scan was performed, this had no discernible effect on image quality.

Two case studies illustrating typical examples are presented below.

\section{Case 1. Angle-closure glaucoma}

A 63-year-old woman presented to the Accident and Emergency Department with a red, painful left eye and reduced vision. The cornea was too oedematous to enable gonioscopic viewing. Optical coherence tomography was performed and revealed a closed angle, confirming the diagnosis of acute angle-closure glaucoma (Fig. 1C). After intraocular pressure (IOP) had been lowered medically, YAG laser peripheral iridotomy (LPI) was performed, with resolution of clinical symptoms. Repeat OCT immediately after the LPI revealed widening of the ACA (Fig. 1D).

\section{Case 2. Plateau iris configuration}

A 56-year-old woman presented to the A \& E Department with a red, painful left eye, headache and nausea. She had no previous ocular or systemic history. Intraocular pressure was $12 \mathrm{mmHg}$ and $48 \mathrm{mmHg}$ in the right and left eyes, respectively. The cornea was oedematous in the left eye and the pupil mid-dilated and fixed. Left acute angle-closure glaucoma was diagnosed and LPI was performed, following administration of intravenous acetazolamide, topical pilocarpine, levobunolol and apraclonidine, and eventually intravenous mannitol. The IOP remained in the 40-49-mmHg range, despite a patent LPI. Imaging of the anterior chamber by OCT showed that the iris root was angulated and iris configuration was compatible with that of plateau iris configuration in both eyes (Fig. 1E). Ultrasound biomicroscopy was performed for comparison, confirming the diagnosis already made by OCT (Fig. 2). Iridoplasty was performed, with normalization of IOP and resolution of symptoms (Fig. 1F). 


\section{Discussion}

Until recently, assessment of the ACA has been limited to subjective procedures, primarily gonioscopy. This method has several disadvantages (Bruno \& Alward 2002): it is commonly regarded as a difficult skill to acquire; accurate interpretation depends on the level of experience of the clinician, and the direct contact with the patient makes it dependent on patient co-operation.

Previous reports of anterior segment OCT were disappointing, with prototype units using a light source wavelength of $0.8 \mu \mathrm{m}$, which was sub-optimal for imaging the actual ACA due to poor penetration of the sclera (Hoerauf et al. 2000; Wirbelauer et al. 2005). Subsequent studies have used OCT units with 1.3- $\mu \mathrm{m}$ wavelength light sources, providing higher resolution images with greater scan and tissue penetration (Radhakrishnan et al. 2005a, 2005b; Wirbelauer et al. 2005). The ability of the commercially available Zeiss Stratus OCT (0.8- $\mu$ m wavelength light source) to visualize ACA configurations and their correlation with the underlying pathophysiology was recently demonstrated in three subjects with impending angle closure, plateau iris configuration and phacomorphic glaucoma, respectively (Leung et al. 2005). This disparity led us to investigate the ability of OCT using a $0.8-\mu \mathrm{m}$ wavelength light source to image the ACA of patients with different angle configurations. Our study suggests that the Zeiss Stratus OCT may be a helpful tool in the assessment of the ACA.

In our study, the Stratus OCT produced clear cross-sectional images of the ACA in all patients with narrow or closed angles and in one of five patients with plateau iris configuration. The iris configuration (as opposed to the angle itself) was clearly demonstrated in all 26 eyes. Although the ACA was not directly visualized in all patients with open angles and most of the patients with plateau iris configuration, the configuration of the peripheral iris in relation to the cornea could be seen, suggesting an open angle in these patients. Our finding that the angle itself could not be visualized in patients with open angles is consistent with previous studies and is due to poor penetration of the sclera by OCT with a $0.8-\mu \mathrm{m}$ wavelength light source (Hoerauf et al. 2000; Wirbelauer et al. 2005).

In patients with plateau iris configuration, the clear demonstration of the iris configuration by OCT meant that non-glaucoma specialists found it easier to make a diagnosis. This is clearly demonstrated in case 2 , in which a patient with plateau iris configuration was initially misdiagnosed with angle-closure glaucoma.

The presence of corneal oedema, which impaired viewing of the ACA by gonioscopy, appeared to have little effect on the ability of OCT to image the ACA as it was clearly demonstrated in all patients with acute angle-closure glaucoma. A limitation of the Stratus OCT is that the software is designed for evaluating the optic nerve head, retinal nerve fibre layer and retinal thickness. Quantitative analysis of the ACA is not possible using the current software.

Optical coherence tomography using a $1.3-\mu \mathrm{m}$ wavelength light source designed specifically for imaging the ACA is now commercially available (e.g. Zeiss Visante OCT; Carl Zeiss Meditec). However, current OCT units using a $0.8-\mu \mathrm{m}$ wavelength light source, available in many eye departments, are able to visualize the anterior chamber configuration in sufficient detail to assist with the everyday clinical assessment of glaucoma patients and may have a role in the teaching of angle assessment to doctors in training.

Stratus OCT would appear to offer a non-contact, convenient and rapid method of assessing the configuration of the anterior chamber. Despite the limitations, it may be of help during the routine clinical assessment and treatment of patients with narrow or closed angles, particularly when gonioscopy is not possible or is difficult to interpret. 


\section{References}

Bruno CA, Alward WL. Gonioscopy in primary angle-closure glaucoma. Semin Ophthalmol. 2002; 17:59-68. [PubMed: 15513458]

Chan RY, Smith JA, Richardson KT. Anterior segment configuration correlated with Shaffer's grading of anterior chamber angle. Arch Ophthalmol. 1981; 99:104-107. [PubMed: 7458734]

Hoerauf H, Wirbelauer C, Scholz C, Engelhardt R, Koch P, Laqua H, Birngruber R. Slit-lamp-adapted optical coherence tomography of the anterior segment. Graefes Arch Clin Exp Ophthalmol. 2000; 238:8-18. [PubMed: 10664046]

Leung CK, Chan WM, Ko CY, Chui SI, Woo J, Tsang MK, Tse RK. Visualization of anterior chamber angle dynamics using optical coherence tomography. Ophthalmology. 2005; 112:980-984. [PubMed: 15936438]

Puech M, El Maftouhi A. Exploration du segment anterieur par OCT 3. J Fr Ophtalmol. 2004; 27:459_ 466. [PubMed: 15179301]

Radhakrishnan S, Goldsmith J, Huang D, Westphal V, Dueker DK, Rollins AM, Izatt JA, Smith SD. Comparison of optical coherence tomography and ultrasound biomicroscopy for detection of narrow anterior chamber angles. Arch Ophthalmol. 2005a; 123:1053-1059. [PubMed: 16087837]

Radhakrishnan S, Huang D, Smith SD. Optical coherence tomography imaging of the anterior chamber angle. Ophthalmol Clin N Am. 2005b; 18:375-381. [PubMed: 16054995]

Riley SF, Nairn JP, Maestre FA, Smith TJ. Analysis of the anterior chamber angle by gonioscopy and by ultrasound biomicroscopy. Int Ophthalmol Clin. 1994; 34:271-282. [PubMed: 7960522]

Shaffer RN, Tour RL. A comparative study of gonioscopic methods. Trans Am Ophthalmol Soc. 1955; 53:189-208. [PubMed: 13360875]

Spaeth GL, Aruajo SV, Azuara-Blanco A. Comparison of the configuration of the human anterior chamber angle as determined by the Spaeth gonioscopic grading system and ultrasound biomicroscopy. Trans Am Ophthalmol Soc. 1995; 93:337-351. [PubMed: 8719685]

Wirbelauer C, Karandish A, Haberle H, Pham DT. Non-contact goniometry with optical coherence tomography. Arch Ophthalmol. 2005; 123:179-185. [PubMed: 15710813] 

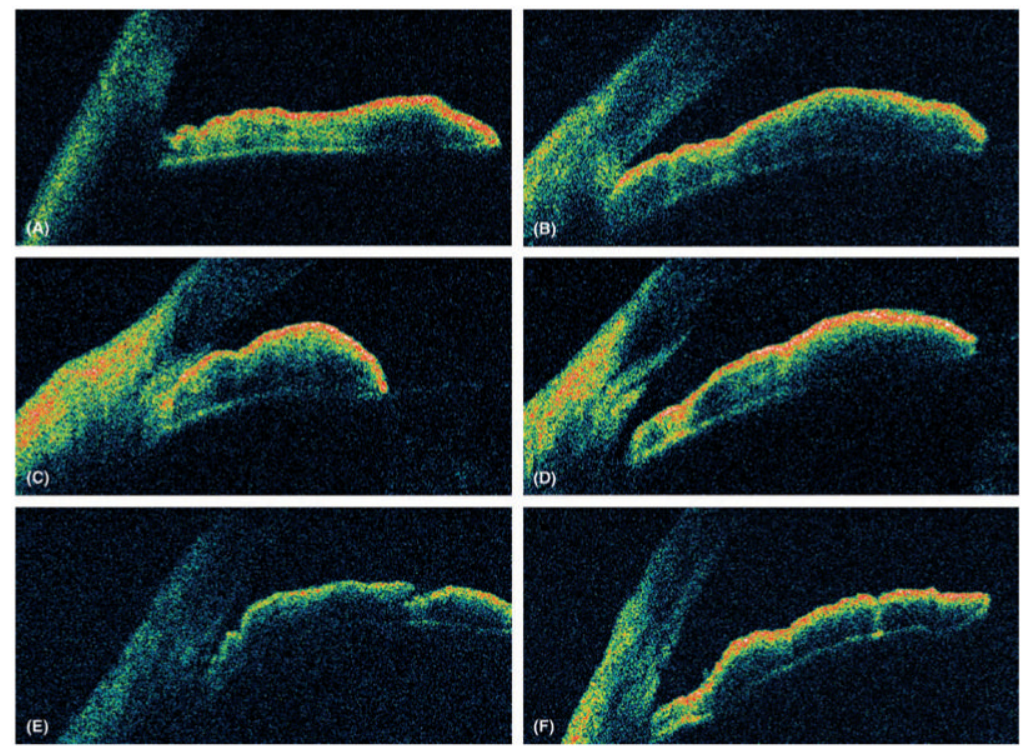

Fig. 1.

Optical coherence tomography images. (A) Normal anterior chamber angle (ACA) configuration. (B) Narrow ACA. (C) Acute angle closure before YAG laser peripheral iridotomy (LPI). (D) Acute angle closure after YAG LPI. (E) Plateau iris. (F) Plateau iris after argon laser iridoplasty. 


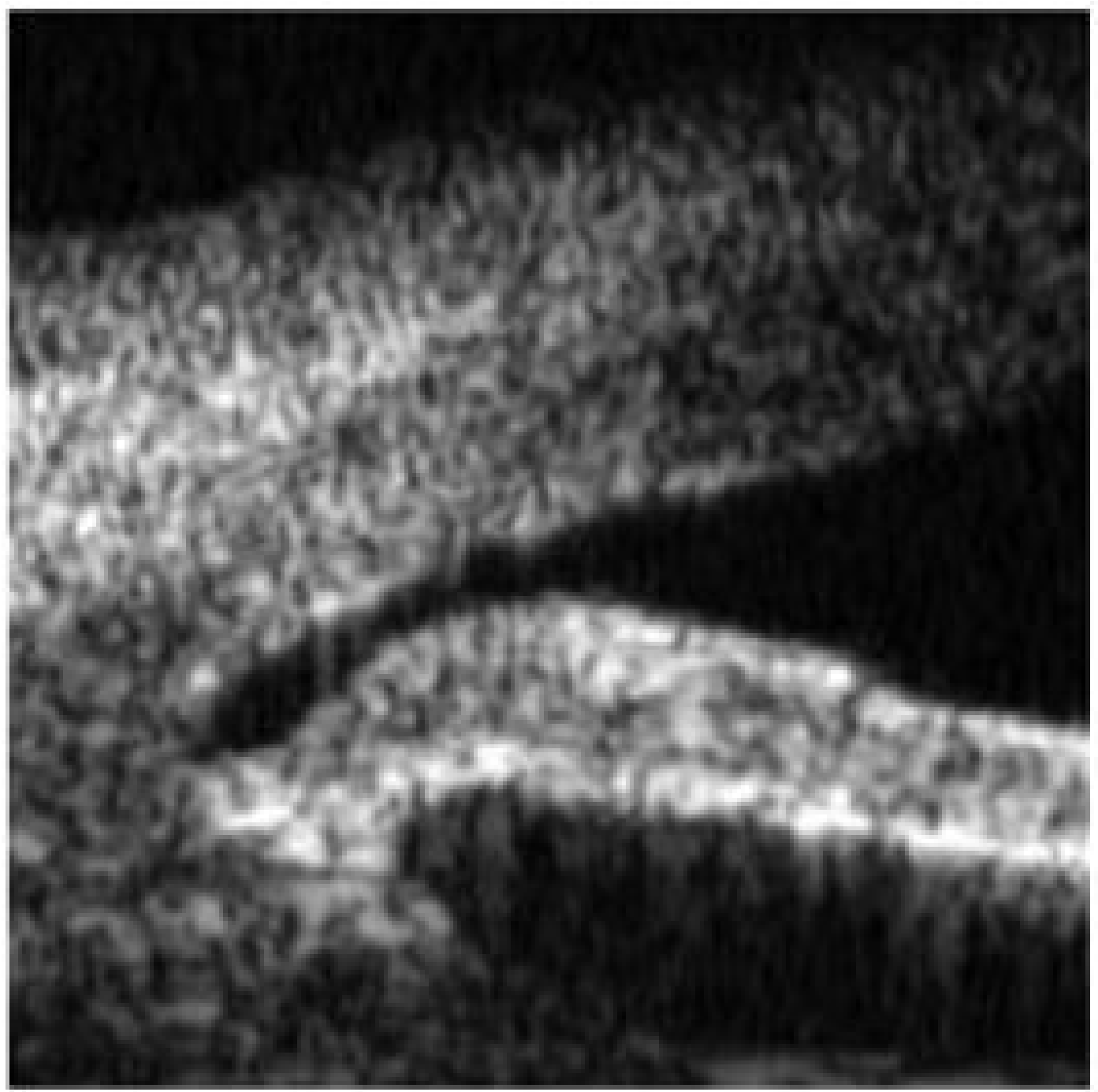

Fig. 2.

Ultrasound biomicrosopy confirming plateau iris. 


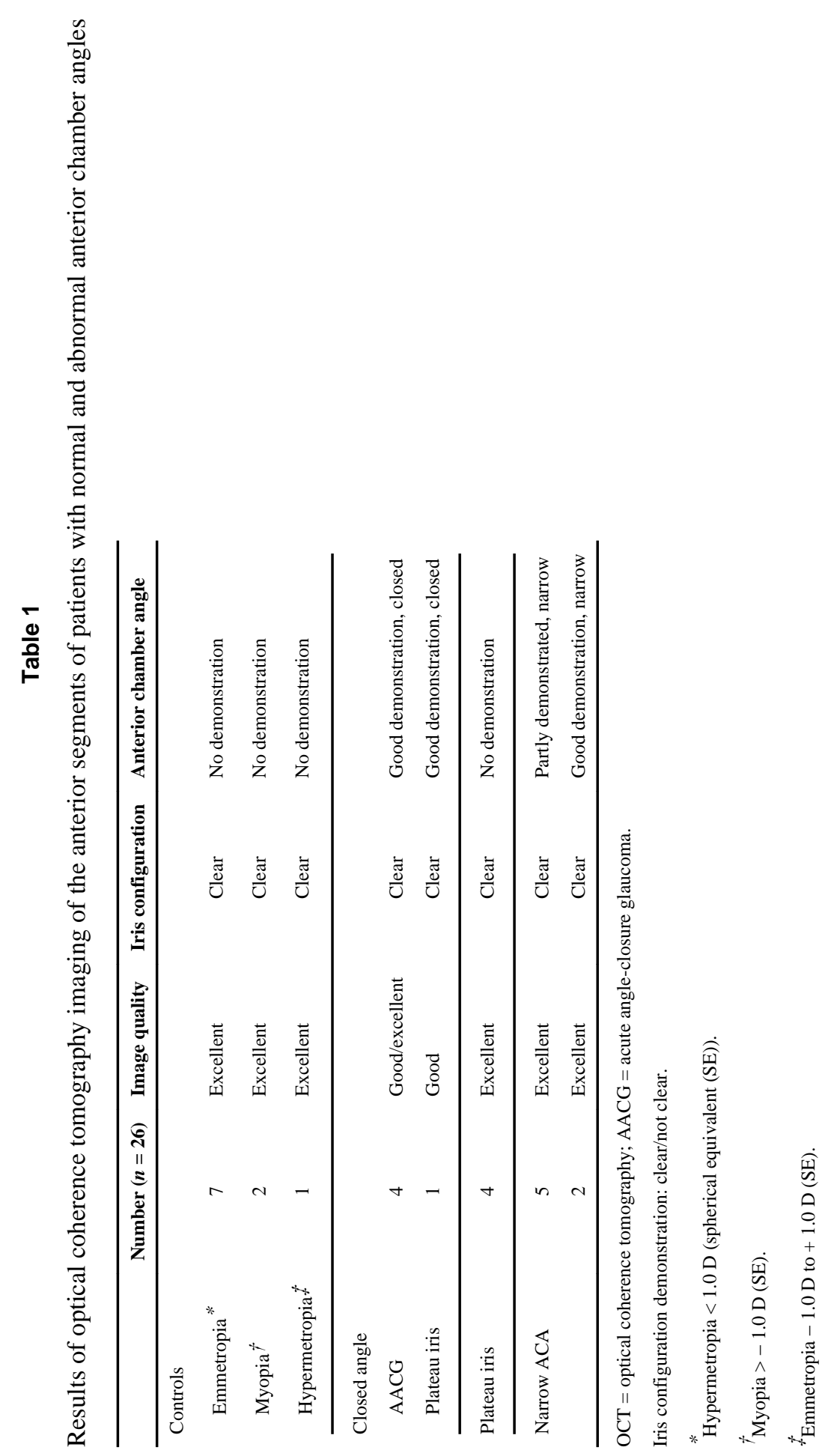

